I BIENAL LATINOAMERICANA DIDH

\title{
El Acuerdo de París sobre cambio climático y el proyecto de la ley marco en Chile: Entre el protagonismo y la denegación de los pueblos indígenas
}

\author{
The Paris Agreement on climate change \\ and the framework law draft in Chile: \\ Between protagonism and denial of indigenous peoples
}

\section{Salvador Millaleo iD \\ Universidad de Chile}

RESUMEN Esta investigación se refiere al rol de los pueblos indígenas en el escenario mundial en las estrategias para enfrentar el cambio climático, debido a sus conocimientos ecológicos tradicionales, como ha sido consagrado en el Acuerdo de París, y que se ha reflejado en leyes nacionales en América Latina como las de México, Guatemala y Perú. Sin embargo, Chile lleva adelante un proyecto de ley marco sobre cambio climático que niega ese rol para los pueblos indígenas.

PALABRAS CLAVE Cambio climático, Acuerdo de París, pueblos indígenas, ley marco.

ABSTRACT This paper refers to the role of indigenous peoples on the world stage in strategies to confront climate change, due to their traditional ecological knowledge, as enshrined in the Paris Agreement, and which has been reflected in national laws in Latin America, for example in Mexico, Guatemala and Peru. However, Chile is currently carrying out a framework law draft on climate change that denies that role for indigenous peoples.

KEYWORDS Climate change, Paris Agreement, indigenous peoples, framework law. 


\section{Introducción}

Este texto analiza cómo el Acuerdo de París sobre Cambio Climático ha establecido nuevas pautas respecto del rol de los pueblos indígenas en las estrategias para enfrentar el cambio climático en el mundo. Se presentan los motivos de por qué los pueblos indígenas buscaron tener protagonismo en este nuevo régimen internacional a partir de la lucha por el reconocimiento de la relevancia de los conocimientos ecológicos tradicionales de los pueblos indígenas. Por otra parte, se analizan los cambios más importantes que dicho nuevo régimen internacional ha significado para la implementación de estrategias estatales de cambio climático en América Latina, en especial en México, Guatemala y Perú. Por último, se analiza cómo la estrategia de Chile, a través del proyecto de ley marco sobre cambio climático, rompe con esas pautas y da la espalda a la participación de los pueblos indígenas.

\section{El Acuerdo de París}

La Conferencia de las Naciones Unidas sobre Cambio Climático 2015 (COP21) fue celebrada junto a la Undécima Conferencia de las Partes del Protocolo de Kioto (CMP11), en París, entre el 30 de noviembre y el 11 de diciembre de 2015. El resultado de dicha conferencia fue el Acuerdo de París.

El Acuerdo de París o Convención Marco sobre el Cambio Climático señala, dentro de sus consideraciones:

Reconociendo que el cambio climático es un problema de toda la humanidad y que, al adoptar medidas para hacerle frente, las partes deberían respetar, promover $\mathrm{y}$ tener en cuenta sus respectivas obligaciones relativas a los derechos humanos, [...] los derechos de los pueblos indígenas.

Y luego:

Observando la importancia de garantizar la integridad de todos los ecosistemas, incluidos los océanos, y la protección de la biodiversidad, reconocida por algunas culturas como la Madre Tierra, y observando también la importancia que tiene para algunos del concepto de «justicia climática», al adoptar medidas para hacer frente al cambio climático.

En el articulado del Acuerdo se incluyó un reconocimiento de los conocimientos tradicionales de los pueblos indígenas:

Las partes reconocen que la labor de adaptación debería llevarse a cabo mediante un enfoque que deje el control en manos de los países, responda a las cuestiones de género

1. Convención Marco sobre el Cambio Climático, 12 de diciembre de 2015, FCCC/CP/2015/L.9. 
y sea participativo y del todo transparente, tomando en consideración a los grupos, comunidades y ecosistemas vulnerables, y que dicha labor debería basarse e inspirarse en la mejor información científica disponible $y$, cuando corresponda, en los conocimientos tradicionales, los conocimientos de los pueblos indígenas y los sistemas de conocimientos locales, con miras a integrar la adaptación en las políticas y medidas socioeconómicas y ambientales pertinentes, cuando sea el caso (artículo 7.5, el énfasis es nuestro).

En la misma conferencia $\mathrm{COP}_{21}$ hubo una alta visibilidad de los pueblos indígenas, facilitada por la concurrencia de más de 160 indígenas representativos a París para participar en las negociaciones, así como la existencia de un pabellón de los pueblos indígenas y una ceremonia de entrega de alto perfil del Premio Ecuatorial 2015 para resaltar la gestión indígena en los asuntos climáticos. Esto fue acompañado por una estrategia mundial de comunicaciones que remarcó la importancia global de los pueblos indígenas y sus derechos para enfrentar el cambio climático. ${ }^{2}$

Durante la $\mathrm{COP}_{21}$, los representantes indígenas señalaron cuatro grandes ejes de problemas relativos al cambio climático (Azpiroz, 2018: 227-228):

- Falsas soluciones: Basadas en mecanismos de mercado que presentan los países desarrollados, las multinacionales y las corporaciones, a las que se dan subsidios masivos y producen más víctimas, debido a los efectos adversos sobre los derechos humanos, el aspecto social, el uso de la tierra, la biodiversidad y los ecosistemas.

- El extractivismo: Los pueblos indígenas denuncian las actividades de empresas extractivas respecto de los recursos naturales y sus consecuencias en cuando a la contaminación, degradación de los suelos y otros elementos de la biodiversidad, enfermedades, crisis social y abusos hacia los pueblos indígenas.

- Falta de reconocimiento oficial y protección integral de los territorios indígenas: Se requiere el reconocimiento completo de la ocupación de un territorio ancestral y su uso colectivo por parte de cada pueblo indígena. Se exige la aplicación del derecho la autodeterminación, de acuerdo con la Declaración de Naciones Unidas sobre los Derechos de los Pueblos Indígenas, el Convenio 169 de la Organización Internacional del Trabajo y la jurisprudencia del Sistema Interamericano de Derechos Humanos.

- No inclusión en el artículo 2 del Acuerdo de París de los derechos humanos y los derechos de los pueblos indígenas, así como de la equidad de género y los derechos de los trabajadores.

2. «Reflexión de la participación de los pueblos indígenas en la COP y las implicaciones del acuerdo de París», Foro Internacional de los Pueblos Indígenas sobre Cambio Climático, 11 de mayo de 2016, disponible en https://bit.ly/2XMhKDS. 
Los resultados del Acuerdo de París fueron magros para los pueblos indígenas, pues no hubo un compromiso fuerte con sus derechos humanos. Según Adelman (2018: 19), el Acuerdo proporcionó una victoria marginal para quienes abogan por la construcción de puentes entre el régimen climático y el derecho de los derechos humanos. A pesar de ello, es claro que el Acuerdo de París hace varias referencias a los pueblos indígenas y afirma la necesidad de reconocer y respetar sus derechos, a diferencia del Protocolo de Kioto, que no hizo ninguna referencia.

Ahora bien, en el Acuerdo de París no se incluyó lo dispuesto en la Declaración de Río sobre el Medio Ambiente y el Desarrollo, cuyo Principio 22 destaca el rol fundamental de los pueblos indígenas y sus comunidades en el manejo del medio ambiente y en el desarrollo gracias a sus conocimientos y prácticas tradicionales.

Asimismo, el Convenio de la Diversidad Biológica, en su artículo 8 letra j), reconoció en forma expresa el papel para la conservación de la diversidad biológica del conocimiento, las prácticas e innovaciones tradicionales de los pueblos indígenas.

En la Conferencia Mundial de los Pueblos sobre el Cambio Climático y los Derechos de la Madre Tierra de Cochabamba en 2010, se había acordado lo siguiente:

Exigimos a los Estados que reconozcan, respeten y garanticen la efectiva aplicación de los estándares internacionales de derechos humanos y los derechos de los pueblos indígenas, en particular la Declaración de las Naciones Unidas sobre los Derechos de los Pueblos Indígenas, el Convenio 169 de la OIT, entre otros instrumentos pertinentes, en el marco de las negociaciones, políticas y medidas para resolver los desafíos planteados por el cambio climático. En especial, demandamos a los Estados a que reconozcan jurídicamente la preexistencia del derecho sobre nuestros territorios, tierras y recursos naturales para posibilitar y fortalecer nuestras formas tradicionales de vida y contribuir efectivamente a la solución del cambio climático.

Demandamos la plena y efectiva aplicación del derecho a la consulta, la participación y el consentimiento previo, libre e informado de los pueblos indígenas en todos los procesos de negociación, así como en el diseño e implementación de las medidas relativas al cambio climático. ${ }^{3}$

En los procesos internacionales de negociación, los pueblos indígenas habían logrado ser considerados una circunscripción dentro de la Conferencia de las Naciones Unidas sobre Cambio Climático en 2001 y como uno de los grupos de interés, con la capacidad de tener reuniones en la sede de la COP, en la forma de un caucus, y presentar declaraciones a las partes (Delgado, 2019). A partir del año 2000, funciona el Foro Internacional de los Pueblos Indígenas sobre Cambio Climático (IIPFCC), establecido como caucus participando en las reuniones COP desde 2008. Un logro de

3. «Conferencia Mundial de los Pueblos sobre el Cambio Climático y los Derechos de la Madre Tierra», Organización de Estados Iberoamericanos, disponible en https://bit.ly/2DQwDhc. 
la COP23 en Bonn en 2017 fue la conformación oficial de la plataforma de los pueblos indígenas, cuyo propósito es reforzar los conocimientos, las tecnologías, las prácticas y los esfuerzos de las comunidades locales y los pueblos indígenas para hacer frente al cambio climático, a fin de facilitar el intercambio de experiencias, mejores prácticas y lecciones aprendidas sobre la mitigación y la adaptación al cambio climático, a fin de mejorar la participación de pueblos indígenas y comunidades locales en los procesos de las COP.

Esta inclusión creciente ha sido un gran logro, toda vez que, a pesar del reconocimiento de sus derechos políticos, territoriales y culturales en el ámbito de Naciones Unidas, y de su posición única para contrarrestar el cambio climático, los pueblos indígenas habían tendido a ser excluidos de las arenas internacionales de discusión y decisión sobre las medidas e instrumentos para mitigar el cambio climático. La exclusión política, económica y epistemológica de los pueblos indígenas ha demorado su protagonismo en dichas discusiones (Comberti y otros, 2019).

Los pueblos indígenas habían participado intensamente -aunque con enfoques críticos- en la iniciativa REDD,$+{ }^{4}$ ya desde la cumbre de Bali de 2007. Esta iniciativa se orientaba hacia políticas e incentivos positivos con el objetivo de reducir las emisiones producto de la deforestación en los países en desarrollo, apoyando la función de conservación, la gestión sostenible de los bosques y el aumento de las reservas forestales de carbono. Estos enfoques fueron denunciados por los pueblos indígenas, ya que, al dar valor monetario a los bosques, han producido conflictos entre comunidades indígenas y el Estado, con exclusión de poblaciones locales de los procesos de implementación y de los beneficios, e incluso expulsiones de sus tierras.

En la actualidad, los pueblos indígenas son formalmente reconocidos dentro de las negociaciones respecto del cambio climático en Naciones Unidas como actores políticos que desempeñan tres papeles: poseedores de conocimiento, parte interesada especialmente vulnerable y protectores de la diversidad biológica, debido a su estrecha relación con la naturaleza (Lindroth y Sinevaara-Niskanen, 2013).

\section{Conocimientos indígenas ecológicos y cambio climático}

Los pueblos indígenas están en el epicentro de los efectos y de las soluciones disponibles respecto del cambio climático (Pearl, 2018: 724 y ss.). Por una parte, constituyen un grupo en especial vulnerable ante los efectos del cambio climático. Esto se debe a que las formas de vida propia de los pueblos indígenas están integradas en los ecosistemas que habitan, con lo que mantienen una relación de estrecha interdependencia con el medio ambiente.

4. Reducción de las Emisiones de la Deforestación y la Degradación de Bosques, disponible en https://redd.unfecc.int. 
Lo anterior hace que los pueblos indígenas sean en particular vulnerables a los impactos del calentamiento antropogénico sobre el medio ambiente, debido a las historias de explotación de su entorno y el despojo colonial que han sufrido, así como por la irreversibilidad del daño climático. La deforestación amenaza los medios, la alimentación, el agua, la salud y la vida, intensifica la pérdida de territorios tradicionales y socava el acceso, uso y propiedad de la tierra y los recursos naturales. La contaminación de los mares y la sobreexplotación de la fauna marina afecta a las comunidades ribereñas que dependen de formas artesanales de pesca; la escasez de agua deteriora la habitabilidad en territorios indígenas (Abate y Kronk, 2013). La pérdida o alteración en los recursos de caza, pesca y agricultura tienen impactos negativos para la seguridad alimentaria y las formas de vida de los pueblos indígenas.

Las áreas administradas por los pueblos indígenas y las comunidades locales se enfrentan a una creciente extracción de recursos, producción, minería y transporte e infraestructura energética, con consecuencias para la subsistencia y salud local; incluso algunas políticas de mitigación del cambio climático tienen impactos negativos sobre los indígenas. Los impactos negativos incluyen la pérdida continua de subsistencia y los medios de vida tradicionales como resultado de la deforestación, la pérdida de humedales, la minería, la propagación de prácticas insostenibles de agricultura, silvicultura y pesca e impactos en la salud y el bienestar por la contaminación y la inseguridad del agua (IPBES, 2019: 14).

Por otra parte, el conocimiento íntimo y de largo aliento del entorno permite a los pueblos indígenas mantener formas de vida autosuficientes a largo plazo, con sistemas de gestión local mejor sintonizados, de manera que pueden ser considerados verdaderos «salvadores del clima» (Zvouloun, 2019). En efecto, las filosofías de los pueblos indígenas consideran a la naturaleza como un complejo integral y holístico, del cual forman parte de manera interrelacionada, interdependiente, con obligaciones y derechos recíprocos entre sí, de las cuales derivan su rol de guardadores y defensores del mundo natural (Galdámez y Millaleo, 2020).

La naturaleza gestionada por los pueblos indígenas y las comunidades locales está bajo presión creciente. Sin embargo, y aunque la diversidad biológica está disminuyendo en el mundo, en la práctica esto sucede con menos rapidez en las tierras de los pueblos indígenas (IPBES, 2019: 14). A pesar de una larga historia de uso de recursos, conflictos de conservación relacionados con la expansión colonial y apropiación de tierras indígenas para parques y usos económicos privados, los pueblos indígenas han manejado sus paisajes terrestres y marinos en formas que se ajustaron a las condiciones locales durante generaciones. Sus métodos de gestión a menudo permanecen compatibles y apoyan en forma activa la conservación de la biodiversidad (IPBES, 2019: 31).

Para el informe de la Plataforma Intergubernamental Científico-normativa sobre Diversidad Biológica y Servicios de los Ecosistemas (IPBES, por sus siglas en inglés), 
una estrategia efectiva para confrontar el cambio climático consiste en reconocer el conocimiento, innovaciones, prácticas, instituciones y valores de los pueblos indígenas, y asegurar su inclusión y participación en la gobernanza del medio ambiente. Esto a menudo mejorará su calidad de vida y las posibilidades de conservación, restauración y uso sostenible de la naturaleza (IPBES, 2019: 18).

Gran parte del mundo silvestre y la biodiversidad se encuentra en áreas tradicionalmente administradas, poseídas, usadas u ocupadas por comunidades indígenas. Éstas han desarrollado formas de domesticación y mantenimiento de variedades locales adaptadas de cultivos, frutas y razas animales; han creado hábitats ricos en especies y alta diversidad de ecosistemas en sus paisajes culturales; han favorecido la identificación de plantas útiles y su cultivo en ecosistemas de alta diversidad; han llevado a cabo una gestión y monitoreo de especies silvestres, hábitats y paisajes para la vida silvestre; han restaurado tierras degradadas; han trabajado en la prevención de la deforestación en territorios indígenas reconocidos, y han ofrecido alternativas para las relaciones entre la humanidad y la naturaleza (IPBES, 2019: 31).

En general, los pueblos indígenas tienen la menor huella ecológica, pero soportan las cargas ambientales más pesadas.

\section{Leyes de cambio climático en América Latina: México, Guatemala y Perú}

A partir de las declaraciones y otros instrumentos adoptados sobre cambio climático a nivel mundial, se han desarrollado marcos legislativos en algunos países para normativizar las estrategias estatales de cambio climático. Según el estudio de Moraga y Meckievi (2016: 6), «si bien las motivaciones son diversas, todas parten de un denominador común: la necesidad de dejar atrás la ambigüedad de instrumentos no vinculantes, como planes y estrategias ministeriales, que poco poder efectivo les otorgan a las autoridades». Los países presentan diversos niveles de avance, con algunos alcanzando logros importantes con su legislación (PNUMA, 2015: 143). Esto es en particular evidente en cuanto a los derechos de los pueblos indígenas. En varias leyes referidas al cambio climático, los pueblos indígenas comienzan a ocupar un rol cada vez más importante. Veremos algunos de estos rasgos en México, Guatemala y Perú.

En México, la Ley General de Cambio Climático, publicada en el Diario Oficial de la Federación el 6 de junio de 2012 (reformada en 2019), señaló de manera escueta en su artículo 26, inciso segundo:

$\mathrm{Al}$ adoptar medidas para hacer frente al cambio climático, se deberán respetar irrestrictamente los derechos humanos, el derecho a la salud, los derechos de los pueblos indígenas, las comunidades locales, los migrantes, los niños, las personas con discapacidad y las personas en situaciones de vulnerabilidad y el derecho al desarrollo, así como la igualdad de género, el empoderamiento de la mujer y la equidad intergeneracional. 
En la ley de Guatemala, Ley Marco para Regular la Reducción de la Vulnerabilidad, la Adaptación Obligatoria ante los Efectos del Cambio Climático y la Mitigación de Gases de Efecto Invernadero (Decreto Legislativo 7-2013), se consideran en el artículo 16 los conocimientos tradicionales, al exigir que las personas individuales o jurídicas, públicas o privadas del país, en la prestación de servicios y producción de bienes, consideren la variabilidad y el cambio climático, así como las condiciones propias de las diferentes regiones, incluidos los conocimientos tradicionales y ancestrales adecuados, aprovechando las tecnologías apropiadas limpias y amigables con el ambiente y con las condiciones ecológicas y biofísicas del país. Así también, deben adoptar nuevas prácticas, incluidas las provenientes del conocimiento tradicional y ancestral, que permitan una producción tal que no se vea afectada por los efectos del cambio climático, garanticen que la población del país cuente con la alimentación básica y minimice los procesos de degradación del suelo. Por otra parte, se contempla la integración de un representante de organizaciones indígenas en el Consejo Nacional de Cambio Climático (artículo 8).

Sin duda, la legislación que más profundiza el rol de los pueblos indígenas frente al cambio climático es la ley marco peruana (Ley 30.754, publicada en el Diario Oficial el 18 de abril de 2018), que fue elaborada considerando una importante participación de las organizaciones indígenas. Una de las características de esta legislación es que atiende de forma diferenciada a los pueblos indígenas y revalora sus prácticas y conocimientos para ayudar a conservar los ecosistemas y a combatir los efectos negativos del cambio climático.

La ley peruana plantea un enfoque integral para la gestión del cambio climático, que debe dialogar, valorar e incorporar las visiones culturales, conocimientos ancestrales y concepciones de bienestar de pueblos indígenas (artículo 3). El artículo 6.6 establece la responsabilidad del Ministerio del Medio Ambiente en promover y llevar a cabo investigación científica y desarrollo tecnológico para la mitigación y adaptación al cambio climático, a través de sus entidades adscritas y especializadas, tomando en consideración los conocimientos tradicionales de los pueblos indígenas u originarios. Así también, el artículo 17.3 señala que los programas y proyectos de conservación y manejo sostenible de bosques incorporen preferentemente prácticas y conocimientos tradicionales de los pueblos indígenas u originarios.

La ley peruana reconoce el papel de los actores no estatales en la lucha contra el cambio climático, en especial la colaboración de los pueblos indígenas, junto con el sector privado y la sociedad civil, para proporcionar recomendaciones relacionadas con las acciones climáticas (Pinto-Bazurco, 2020: 91).

En el artículo 22 de la ley peruana se establece que el Estado salvaguarda el derecho de participación de los pueblos indígenas u originarios, respetando su identidad social, colectiva y cultural, sus costumbres, tradiciones e instituciones, en la formulación, implementación, seguimiento y evaluación de las políticas públicas y proyectos 
de inversión referidos al cambio climático que los afecte, en lo que corresponda al Convenio 169 de la OIT.

El reglamento de dicha ley crea una plataforma climática indígena, la que servirá como un espacio de los pueblos indígenas para la gestión, articulación, intercambio, sistematización, difusión y seguimiento de sus propuestas de medidas de adaptación y mitigación, y de los conocimientos, prácticas y saberes tradicionales y ancestrales en cambio climático que contribuyen a la gestión integral del cambio climático, según el Acuerdo de París.

En el capítulo 4 sobre medidas de mitigación y adaptación, se incorpora la implementación de una educación ambiental con enfoque intercultural.

\section{Proyecto de ley marco de cambio climático en Chile}

El Convenio de París fue ratificado por Chile el 10 de febrero de 2017. A consecuencia de ello, el Gobierno del presidente Sebastián Piñera lanzó una consulta nacional durante 2018, que consistió en un proceso de participación y discusión para la elaboración de un anteproyecto de ley marco de cambio climático para Chile. Durante dicho proceso se celebraron talleres, diálogos y consultas públicas tanto en las regiones como en la capital, Santiago. Estos diálogos ciudadanos se celebraron en las dieciséis regiones del país (Biskupovik, Sepúlveda y Carmona, 2020: 2). Sin embargo, como señala Jessica Cayupi, claramente la convocatoria para los diálogos ciudadanos no se extendió a organizaciones ni comunidades indígenas, ni tampoco se les informó del proceso, con lo que se obvió el derecho de participación consagrado en el Convenio 169 y la obligación correlativa del Estado de asegurarlo (Cayupi, 2019).

Después del proceso participativo referido, el 13 de enero de 2020, el Gobierno ingresó al Congreso Nacional el proyecto de ley marco de cambio climático, Boletín 13.191-12.

El artículo 2 del proyecto de ley señala cuáles son los principios que inspirarán las políticas, planes, programas, normas, acciones y demás instrumentos que se dicten o ejecuten para enfrentar el cambio climático. Dentro de dichos principios se establece el principio de equidad. Por ella, se entiende el deber del Estado de procurar una justa asignación de cargas, costos y beneficios, con enfoque de género y especial énfasis en sectores, comunidades y ecosistemas vulnerables al cambio climático. No se establece allí ninguna referencia especial respecto de las comunidades indígenas.

Uno de los elementos que más resaltan de la norma propuesta consiste en la total ausencia de reconocimiento - y no digamos de protección- de los conocimientos ecológicos tradicionales de los pueblos indígenas.

Por cierto, esto tiene un contexto. En primer lugar, en Chile existe una protección muy débil de los conocimientos tradicionales de los pueblos indígenas, de manera que son apropiados por terceros en diversas formas sin consentimiento de los pue- 
blos que los generaron y los mantienen. Apenas existe una prohibición en la Ley de Propiedad Industrial para impedir la constitución de derechos de patentes respecto de conocimientos tradicionales.

En segundo lugar, la protección de las tierras indígenas es también débil. Las tierras subsistentes están protegidas por la ley indígena (Ley 19.253), pero éstas representan menos de la mitad de las que el Estado tituló para los indígenas a fines del siglo XIX, mientras que las otras pasaron a terceros no indígenas, tanto por vías legales como ilegales. El mecanismo de recuperación de tierras instalado por la ley en 1993 ha operado a través de la compra en el mercado, mediante un sistema engorroso, dilatado y costoso, que solo ha podido recuperar una porción marginal de las tierras perdidas mientras ha operado.

A ello hay que agregar que los pueblos indígenas prácticamente no tienen derechos respecto de las áreas silvestres protegidas por el Estado que habitan. Salvo algunas excepciones, en que parques nacionales son administrados o coadministrados por los pueblos indígenas (Rapa Nui y Los Flamencos), la casi totalidad de las áreas son administradas por agencias estatales (la Corporación Nacional Forestal, Conaf).

A nivel internacional, se ha sostenido que los derechos de tenencia para los pueblos indígenas claros y seguros son esenciales para la gestión sostenible y la conservación efectiva de los bosques y otros ecosistemas ricos en carbono (turberas, praderas y humedales costeros). En virtud de esos derechos, los pueblos indígenas ven protegida su capacidad para ejercer su autogobierno, adaptarse a su entorno cambiante y administrar los ecosistemas de los que dependen al incentivar el mantenimiento y la restauración, y para resolver el riesgo de conflicto y apropiación ilegal por parte de otros (RRI, 2019).

Lo anterior nos lleva a precisar, en tercer lugar, que en Chile no se reconoce el derecho al autogobierno de los pueblos indígenas, de manera que sus poderes de gestión son limitados y solo están respaldados cuando tienen títulos de propiedad. Las consultas indígenas, por su parte, y a pesar de la incorporación en 2009 del Convenio 169 de la OIT, en materia ambiental están sumamente restringidas, toda vez que solo se aplican a aquellos proyectos que deben contar con un estudio de impacto ambiental, cuando los impactos posibles se pueden justificar como significativos; además, no son en realidad previos, por lo que operan cuando los proyectos de inversión no admiten con facilidad modificaciones, por lo que limitan solo a la discusión de reparaciones y mitigaciones.

Lo anterior se resume en que Chile es el país más atrasado en la región en materia de reconocimiento y protección de los derechos de los pueblos indígenas, tanto que ni siquiera tienen reconocimiento a nivel constitucional. Ello se expresa en relaciones de tensión entre el Estado y los pueblos indígenas, que se han manifestado en una serie de observaciones de organismos internacionales de derechos humanos 
- en varios ámbitos- por las afectaciones a sus derechos humanos; e incluso condenas, como la del caso Lonkos en la Corte Interamericana de Derechos Humanos en $2014 .^{5}$

Estos factores han sido determinantes en que Chile se margine de las tendencias globales y de los modelos regionales para permitir el necesario protagonismo de los pueblos indígenas en las estrategias de cambio climático.

Sin duda, lo más crítico es la falta de protección de los conocimientos ecológicos tradicionales, lo cual es coincidente con que el proyecto de ley marco chileno no los considere como factores valiosos para su transferencia y aplicación en el resto de la sociedad. Así, tampoco se rescata su importancia para la educación ambiental.

La referencia al respeto a los derechos de los pueblos indígenas no aparece en ninguna parte del proyecto, ni siquiera en su fundamentación. Tampoco hay un enfoque diferenciado para sus especiales condiciones como sujetos vulnerables a los efectos negativos del cambio climático.

Esto se ve acompañado por la falta de participación de los pueblos indígenas en materias de acción climática. En efecto, el artículo 31 del proyecto señala que toda persona o agrupación de personas tendrá derecho a participar, de manera informada, en la elaboración, revisión y actualización de los instrumentos de gestión del cambio climático, mediante los mecanismos provistos para ello en la ley. El proyecto, por cierto, no contempla mecanismos diferenciados de participación de los pueblos indígenas. Con ello, se vulnera el artículo 6 del Convenio 169 de la OIT, que señala que los Estados tienen el deber de establecer los medios a través de los cuales los pueblos interesados puedan participar de manera libre, por lo menos en la misma medida que otros sectores de la población, y a todos los niveles en la adopción de decisiones en instituciones electivas y organismos administrativos y de otra índole responsables de políticas y programas que les concierna.

En ninguna de las instancias de deliberación, consulta y participación que crea el proyecto de ley se incorporan a los pueblos indígenas, como el Comité Científico Asesor para el Cambio Climático y el Consejo Nacional para la Sustentabilidad y el Cambio Climático. Estas omisiones expresan una denegación de los derechos e intereses ambientales de los pueblos indígenas en Chile, $y$ han sido cuestionadas y rechazadas por la lucha de las comunidades para proteger sus ecosistemas y formas de vida.

5. Sentencia del caso Norín Catrimán y otros (dirigentes, miembros y activistas del pueblo indígena mapuche) con Chile, Corte Interamericana de Derechos Humanos, fondo, reparaciones y costas, 29 de mayo de 2014. 


\section{Conclusiones}

El Acuerdo de París reflejó, de una manera pálida, los derechos de los pueblos indígenas y su rol respecto de los efectos del cambio climático, la justicia ambiental y la correspondiente acción climática.

Ello es producto de un protagonismo que han ido tomando los pueblos indígenas en las negociaciones de los foros internacionales, en los que se ha reconocido su rol en el epicentro del cambio climático, como grupos especialmente vulnerables y como portadores de conocimientos tradicionales que permiten una mejor gestión de la diversidad biológica y protección de los ecosistemas en dicho contexto: la salvación climática.

Este proceso, difícil y lleno de límites y obstáculos, se ha reflejado de manera paralela o posterior en procesos estatales en que las legislaciones internas de diversos países procuran reflejar ese protagonismo de los pueblos indígenas en la acción climática. Los casos de México, Guatemala y Perú son muy ilustrativos al respecto.

En contraste con esas tendencias de transformación que provienen de la esfera internacional, Chile muestra un ejemplo disonante de extraña resistencia a los derechos e intereses ambientales indígenas, al denegar su rol en la acción climática. Esto, por cierto, se explica por las particularidades del caso chileno en cuanto al retraso de las relaciones entre el Estado y los pueblos indígenas.

\section{Referencias}

AвAтE, Randall y Elizabeth Kronk (2013). «Commonality among unique indigenous communities: An introduction to climate change and its impacts on indigenous peoples». En Randall Abate y Elizabeth Kronk (editores), Climate change and indigenous peoples: The search for legal remedies (pp. 3-18). Cheltenham: Edward Elgar.

Adelman, Sam (2018). «Human rights in the Paris Agreement: Too little, too late?». Transnational Environmental Law, 7 (1): 17-36. DOI: 10.1017/S2047102517000280. AzPiroz, María (2018). «Actores sociales en la Cumbre del Clima de París: El mensaje de pueblos indígenas, grupos religiosos, mujeres y jóvenes». Revista Mexicana de Ciencias Políticas y Sociales, 63 (233): 221-254. DOI: 10.22201/ fcpys.2448492xe.2018.233.59458.

Biskupovik, Consuelo, Macarena Sepúlveda y Rosario Carmona (2020). Ley Marco de Cambio Climático y pueblos indígenas en Chile: Elementos para su incorporación. Santiago: CIIR.

CAYUPI, Jessica (2019). Pueblos indígenas, actores claves en la gobernanza del cambio climático. Santiago: Observatorio Ley de Cambio Climático para Chile, CR2, Centro de Derecho Ambiental de la Universidad de Chile. 
Comberti, Claudia, Thomas Thornton, Michaela Korodimou, Meghan Shea y Kimaren Ole Riamit (2019). «Adaptation and resilience at the margins: Addressing indigenous peoples' marginalization at International Climate Negotiations». Environment: Science and Policy for Sustainable Development, 61 (2): 14-30. DOI: 10.1080/00139157.2019.1564213.

Delgado, Deborah (2019). La participación de los pueblos indígenas en la Convención Marco de las Naciones Unidas sobre el Cambio Climático: De actores «tradicionales» a actores frente al Antropoceno. Madrid: Fundación Carolina.

Galdámez, Liliana y Salvador Millaleo (2020). «La naturaleza en la Constitución: Visiones indígenas y propuestas ante la crisis». Acta Bioethica, 26 (1): 51-6o. DOI: 10.4067/S1726-569X2020000100051.

IPBES, Plataforma Intergubernamental Científico-normativa sobre Diversidad Biológica y Servicios de los Ecosistemas (2019). Summary for policymakers of the global assessment report on biodiversity and ecosystem services of the Intergovernmental Science-Policy Platform on Biodiversity and Ecosystem Services. Bonn: IPBES Secretariat.

Lindroth, Marjo y Heidi Sinevaara-Niskanen (2013). «At the crossroads of autonomy and essentialism: Indigenous peoples in international environmental politics». International Political Sociology, 7 (3): 275-293. DOI: 10.1111/ips.12023.

Moraga, Pilar y Sol Meckievi (2016). Análisis comparativo de legislación de cambio climático. Santiago: CR2.

Pearl, Alexander (2018). «Human rights, indigenous peoples, and the global climate crisis». Wake Forest Law Review, 53: 713-738. Disponible en https://ssrn.com/ abstract $=3328592$.

Pinto-Bazurco, José (2020). Los retos del cambio climático: Un estudio sobre las respuestas legales del Perú. Lima: Universidad de Lima, Fondo Editorial.

PNUMA, Programa de las Naciones Unidas para el Medio Ambiente (2015). Aportes legislativos de América Latina y el Caribe en materia de cambio climático. Ciudad de Panamá: PNUMA, Parlatino.

RRI, Rights and Resources Initiatives (2019). Secure indigenous peoples and community land rights as a nature-based solution to climate change. Washington D. C.

Zvouloun, Shy (2019). «Indigenous peoples as climate saviours, how defending indigenous rights could save the planet». Leviathan, 10 (1): 20-24.

\section{Sobre el autor}

Salvador Millaleo es abogado, doctor en Sociología de la Universidad de Bielefeld, Alemania. Investigador en el Centro de Derechos Humanos, Facultad de Derecho Universidad de Chile. Su correo electrónico es smillaleo@derecho.uchile.cl. (D) https://orcid.org/000o-0001-6559-4856. 
El Anuario de Derechos Humanos es una publicación semestral de referencia y consulta en materia de derechos humanos y campos afines. Busca ser un espacio de discusión de los temas centrales en el ámbito nacional e internacional sobre derechos humanos. Es publicado desde 2005 por el Centro de Derechos Humanos de la Facultad de Derecho de la Universidad de Chile.

\author{
EDITORA GENERAL \\ Claudia Iriarte Rivas \\ ciriarter@derecho.uchile.cl \\ EDITORA DE ESTE NÚMERO \\ Liliana Galdámez Zelada \\ lgaldamez@derecho.uchile.cl \\ SITIO WEB \\ anuariocdh.uchile.cl \\ CORREO ELECTRÓNICO \\ anuario-cdh@derecho.uchile.cl \\ LICENCIA DE ESTE ARTÍCULO
}

Creative Commons Atribución Compartir Igual 4.o Internacional

\author{
कै \\ La edición de textos, el diseño editorial \\ y la conversión a formatos electrónicos de este artículo \\ estuvieron a cargo de Tipográfica \\ (www.tipografica.io)
}

